\title{
Physical exercise is essential for cardiac autonomic regulation in hypertensive patients treated with losartan or enalapril
}

\author{
Tábata de Paula Facioli \\ University of Sao Paulo Faculty of Medicine of Ribeirao Preto: Universidade de Sao Paulo Faculdade de Medicina de Ribeirao Preto \\ Stella Vieira Philbois \\ University of Sao Paulo Faculty of Medicine of Ribeirao Preto: Universidade de Sao Paulo Faculdade de Medicina de Ribeirao Preto \\ Jens Tank \\ German Aerospace Centre DLR Institute of Aerospace Medicine Site Cologne: Deutsches Zentrum fur Luft- und Raumfahrt DLR Institut fur Luft- und \\ Raumfahrtmedizin \\ Hugo Celso Dutra de Souza ( $\sim$ hugocds@fmrp.usp.br) \\ Universidade de Sao Paulo https://orcid.org/0000-0002-0009-0005
}

\section{Original Research Article}

Keywords: Hypertension, cardiac autonomic control, pharmacological treatment, aerobic physical training

Posted Date: January 28th, 2022

DOI: https://doi.org/10.21203/rs.3.rs-1284632/v1

License: @ (i) This work is licensed under a Creative Commons Attribution 4.0 International License. Read Full License 


\section{Abstract \\ Background}

Hypertension treatment with renin-angiotensin system inhibitors (RASi) presents contradictions in relation to the recovery of damage in cardiovascular autonomic control. Conversely, the association of RASi with aerobic physical exercise can be a determining factor for some gains to be achieved. Thus, the objective was to investigate the effects of aerobic physical training on hemodynamic and cardiac autonomic regulation in hypertensive volunteers untreated and treated with RASi.

\section{Methods}

Fifty-four men ( $\cong 40-60$ years old) with a history of hypertension for more than two years were divided into three groups: untreated (Control; $\mathrm{N}=16$ ), treated with $\mathrm{AT}_{1}$ receptor blocker (losartan; $\mathrm{N}=21$ ), and treated with angiotensin-converting enzyme inhibitor (enalapril; $\mathrm{N}=17$ ). All subjects underwent hemodynamic, metabolic, and cardiovascular autonomic evaluation using baroreflex sensitivity (BRS) and spectral analysis of heart rate variability (HRV) and blood pressure variability (BPV), before and after 16 weeks of supervised aerobic physical training.

\section{Results}

The volunteers treated with RASi had lower BPV and HRV, both in the supine position and in the tilt test, with the losartan group having the lowest values. Aerobic physical training increased HRV and BRS in all groups. However, the association of enalapril with physical training appears to have more prominent results.

\section{Conclusion}

Long-term treatment with enalapril and losartan may have detrimental effects on the autonomic modulation of HRV and BRS. Aerobic physical training is essential to promote positive adjustments in the autonomic modulation of HRV and BRS in hypertensive patients treated with RASi, especially with enalapril treatment. Trial Register. Brazilian Registry of Clinical Trials (RBR-8k2dsc).

\section{Key Points}

- Hypertensive patients have reduced heart rate variability

- Inhibitors of the renin-angiotensin system reduce blood pressure but have little effect on heart rate variability.

- Enalapril maleate has more advantages over autonomic modulation when compared to losartan

- Regular aerobic exercise is important tool for recovering from heart rate variability.

\section{Introduction}

Until a few decades ago, the treatment of patients with systemic arterial hypertension (SAH) consisted of an approach based mainly on pharmacological therapy. Among the different classes of antihypertensive drugs, type 1 angiotensin II (AT 1 ) receptor blockers and angiotensin-converting enzyme inhibitors (ACEi) have been widely used. These drugs are also associated with other positive cardiovascular effects, such as the influence on cardiac autonomic regulation, characterized mainly by a reduction in the sympathetic component [1-3]. However, these benefits seem to be associated with short-and mediumterm treatments, since studies that investigated the long-term effects showed contradictory autonomic results, especially in relation to cardiac autonomic modulation and baroreflex sensitivity (BRS) [2,4-6]. In this case, it is possible that long-term monotherapeutic treatment with renin-angiotensin system inhibitors results in the impairment of cardiac autonomic regulation, despite the normalisation of the blood pressure (BP) $[7,8]$

More recently, cardiovascular physiotherapy through regular practice of physical exercises, was introduced as an adjunct treatment to pharmacological treatment or as the first therapeutic choice before drug prescription, especially in patients with mild to moderate hypertension [9, 10] This therapeutic approach, based mainly on monitored and supervised aerobic physical training, has many benefits such as adaptations in cardiac morphology and functionality, increased endothelial vasodilator response and positive effects on cardiovascular autonomic control [11-17]. Among cardiovascular autonomic effects, there is a reduction in sympathetic autonomic influence on the heart and vessels and an increase in vagal influence on the heart [14, 15, 18]. The cardiovascular autonomic benefits promoted by aerobic physical training seem to be much more robust and less controversial. Our hypothesis is that aerobic physical training is essential to recovery of cardiovascular autonomic regulation in hypertensive patients, regardless the pharmacological treatment. In addition, it is possible that the combination of both treatments has a more prominent effect on cardiovascular autonomic control. This is important because impairment in cardiovascular autonomic regulation is considered a strong predictor of cardiovascular morbidity/mortality [19-21].

Therefore, this study aimed to investigate in volunteers with SAH treated with losartan, an $\mathrm{AT}_{1}$ receptor blocker, and enalapril maleate, an ACEi, influences cardiovascular autonomic control, focusing on BRS and in the modulation of heart rate (HRV) and blood pressure variability (BPV). We also investigated the aerobic physical training effects compared with pharmacological treatment, as well as the effects of the association of both treatments. 


\section{Methods}

\subsection{Sampling}

Fifty-four men, aged between 40 and 60 years, who did not practice regular physical exercises, were studied. These volunteers were divided into three groups (Figure 1); untreated hypertensive (control group; $\mathrm{N}=16$ ), hypertensive treated with losartan (losartan group; $\mathrm{N}=21$ ), and hypertensive treated with enalapril maleate (enalapril group; $\mathrm{N}=17$ ). All volunteers were screened at the Laboratory of Physiology and Cardiovascular Physiotherapy (Laphy-Carphy) of the Ribeirão Preto Medical School (FMRP-USP). The volunteers were diagnosed with SAH for at least 2 years, classified into stages I and II, with low to moderate cardiovascular risk [9]. The treatment was monotherapy with antihypertensive drugs with $\mathrm{AT}_{1}$ receptor blocker (losartan, 25-50 mg/day) or ACEi (enalapril maleate, $10-20 \mathrm{mg} /$ day) for at least six months to two years before being included in the study.

The choice to include only men was based on previous studies, which showed that men and women have differences in cardiac autonomic modulation balance. In this case, the men had a greater predominance of the sympathetic autonomic component [22-24].

None of the volunteers performed regular practice of physical exercise, none presented cognitive disorders, musculoskeletal disorders, endocrine, metabolic and cardiovascular diseases (except SAH), or had any other disease or physical limitation that compromises the performance in the tests. We also did not include smokers or volunteers with SAH who were using combined pharmacological therapy or who used any other medication that interfered with cardiac function and cardiovascular autonomic control.

\subsection{Ethical aspects}

All volunteers were interviewed, in addition to checking their respective medical records that contain all the data related to identification and disease treatment. All study procedures were performed in accordance with the guidelines established in the Declaration of Helsinki and Good Clinical Practice. This study was approved by the Human Research Ethics Committee of Hospital of Ribeirão Preto Medical School, University of São Paulo, Brazil (2.280.910/2017). All volunteers underwent careful evaluation, were informed about the legal and ethical aspects of the study and signed a free and informed consent form before the study started. This study was registered in the Brazilian Registry of Clinical Trials (RBR-8k2dsc).

\subsection{Experimental protocols}

Data were collected during three visits between 7-10 am, with an interval of $48 \mathrm{~h}$ between visits. During the first visit, anthropometric measurements and blood samples were taken to analyse the metabolic profiles at the Laboratory of the Clinical Research Support Centre of the Ribeirão Preto Medical School's Hospital (HCFMRP/USP). During the second visit, the cardiorespiratory functional test was performed, while on the third and last visits, electrocardiographic and pulsatile BP records were performed for the analysis of the cardiovascular autonomic modulation, both at the Laboratory of Physiology and Cardiovascular Physiotherapy (Laphy-Carphy) of the Ribeirão Preto Medical School. Each visit lasted $\sim 2 h$, and all volunteers were instructed not to drink alcohol and caffeine, not perform strenuous physical exercises, and to maintain their usual diet for $48 \mathrm{~h}$ before the evaluations. The volunteers in the drug groups were instructed not to interrupt the pharmacological treatment on their own and were advised to sleep at least 7 or $8 \mathrm{~h}$ on the night before the visits.

The protocols were repeated after 16 weeks of supervised aerobic physical training on a motorised treadmill. In addition, for the final evaluation, an interval of 48h was established between the last session of physical training and the first day of revaluation.

\subsection{Anthropometric parameters}

Body weight and height were obtained using an analogue scale with an altimeter (Welmy), while the body mass index (BMI) values were obtained using the formula $\mathrm{W} / \mathrm{H}^{2}$, where $\mathrm{W}$ is the weight in kilogrammes and $\mathrm{H}$ is the height of the subject in metres. Body composition was evaluated using the bioelectrical impedance method (Quantum BIA 101; Q-RJL Systems, Clinton Township, Michigan, USA).

\subsection{Laboratory exams}

Blood samples (3.5 mL, BD Vaccutainer® EDTA - Becton, Dickin, and Company, Franklin Lakes, NJ, USA) were used to analyse the fasting glycaemia (hexoquinase-UV), triglycerides (desidrogenase), and total cholesterol and fractions (esterase-oxidase). All volunteers were asked to fast for 12 hours prior to the assessments.

\subsection{Cardiorespiratory Function Test}

An incremental treadmill exercise test was performed along with a submaximal test, established with the heart rate (HR) corresponding to the sum of the baseline HR and $85 \%$ of the reserve HR (maximum HR - basal HR), following the previously described Balke protocol [25]. Electrical activity was monitored by an electrocardiogram (ECG) with one lead (CM5). Oxygen and dioxide carbon uptake $\left(\mathrm{VO}_{2}\right.$ and $\left.\mathrm{VCO}_{2}\right)$ were obtained using a metabolic analyser (Ultima ${ }^{\mathrm{TM}}$ CardiO $_{2}$, Medical Graphics Corp., St. Paul, Minneapolis, USA).

\subsection{Heart Rate Variability and Blood Pressure Variability Analysis}

The spectral analysis of HRV was recorded between 8 am and 10 am according to the following protocol: after remaining in a supine rest position on an orthostatic bed for $20 \mathrm{~min}$, the volunteers were head up tilted passively $\left(75^{\circ}\right.$ angle) for an additional 10 min. HRV for the supine and head up tilted positions (i.e., tilt test) was recorded using an electrocardiogram (ADInstruments, Bella Vista, Australia), and a time series of RR interval (RRi) was determined. The HRV was obtained using the RRi from the electrocardiographic record (ECG) through the modified CM5 shunt at a sampling frequency of $1000 \mathrm{~Hz}$. The BPV data values were obtained from the systolic BP (SBP) recorded beat-to-beat using the digital plethysmography recording equipment (Finometer Pro, Finapress 
Medical System, Amsterdam, Netherlands) using a cuff positioned on the middle finger of the right upper arm. The BPV and HRV analyses were performed as previously described using custom computer software (CardioSeries v2.7, http://sites.google.com/site/cardioseries) [22, 23, 26, 27].

\subsection{Spontaneous baroreflex sensitivity}

BRS was assessed in the time domain using the sequence technique. The computer software CardioSeries v2.7 scanned the beat-to-beat time series of pulse interval (PI) and SBP values, searching for sequences of at least 3 consecutive beats, in which progressive increases in SBP were followed by progressive increases in $\mathrm{PI}$ (up sequence) and progressive decreases in SBP were followed by progressive decreases in PI (down sequence), with a correlation coefficient ( $r$ ) between PI and SBP (values higher than 0.8). Spontaneous BRS was determined by the mean slope of the linear regression line between the SBP and PI values of each sequence. The number of baroreflex sequences found (per 1000 beats) and the mean individual slope of the significant SBP/PI relationship, obtained by averaging all slopes computed within the test period, were calculated, and used as a measure of spontaneous BRS [23, 26, 28, 29].

The sequence method also presents the baroreflex effectiveness index (BEI). It is the ratio of the number of sequences and the total number of SBP ramps. The BEl shows how many SBP changes are effectively translated into a change in PI, independent of its magnitude.

\subsection{Aerobic physical training}

The sessions were supervised, monitored, and performed three times per week for 16 weeks. The training intensity was calculated as the sum of HR at rest and $70-80 \%$ of reserve HR, obtained by means of the following equation: HR recorded at the peak of the cardiopulmonary testing - HR at rest. The training sessions lasted for $1 \mathrm{~h}$, divided into three phases as follows: 5 min of warm-up using an intensity lower than the target $\mathrm{HR}$ training range (50-65\% of reserve $\mathrm{HR}$ ), 50 min of training using the training HR (70-80\% of reserve HR) and 5 min of cool-down using an intensity lower than the training HR (40-50\% of reserve HR). There was an adaptation period during the first two weeks of the study, during which participants went through 20-30-min sessions for familiarization and adaptation to the training protocol. The intensity used was equivalent to the sum of HR at rest and $50 \%-60 \%$ of the reserve HR, followed by increases in the intensity and duration in the subsequent weeks, until the volunteers reached the training HR as described. HR was monitored throughout the sessions using a pulse frequency meter (Polar RS810).

\subsection{Statistical analysis}

The results are presented as mean \pm standard deviation (SD). Variables were analysed using parametric and nonparametric tests, when required. Age and height were assessed using one-way analysis of variance (ANOVA). When appropriate, post hoc comparisons were performed using Tukey's test. The effects of laboratory examinations, hemodynamic parameters, cardiopulmonary functional tests, HRV, BPV, and spontaneous BRS were assessed by two-way ANOVA. When appropriate, post hoc comparisons were performed using the Student-Newman-Keuls method. Student's t test was used for comparisons between groups, and paired Student's t test for intragroup comparison (before and after training). Differences were considered significant at $P<0.05$. All statistical tests were performed using SigmaPlot 11.0 software (Systat Software Inc., San Jose, CA, USA). The SigmaPlot 11.0 software was used for sample size calculation, confidence level was set at $95 \%$, and power at $80 \%$, with the LF and HF variables in normalized units. The sample size was set to 16 participants per group.

\section{Results}

The BP and HR values are presented in Table 1. The control group had lower HR values and higher BP values than the losartan and enalapril groups. After aerobic physical training for 16 weeks, all groups showed similar values, characterized by a reduction in baseline HR and BP. 
Table 1

Anthropometric, hemodynamic, and metabolic parameters of all groups, before and after the 16-week aerobic physical training.

\begin{tabular}{|c|c|c|c|c|c|c|c|c|c|}
\hline & \multicolumn{3}{|c|}{ Before Training } & \multicolumn{3}{|c|}{ After training } & \multirow{2}{*}{$\begin{array}{l}\text { Drug } \\
\text { Factor } \\
\text { P }\end{array}$} & \multirow{2}{*}{$\begin{array}{l}\text { Training } \\
\text { Factor } \\
\text { P }\end{array}$} & \multirow{2}{*}{$\begin{array}{l}\text { Interaction } \\
\mathrm{P}\end{array}$} \\
\hline & Control & Losartan & Enalapril & Control & Losartan & Enalapril & & & \\
\hline \multicolumn{10}{|l|}{$\begin{array}{l}\text { Anthropometric } \\
\text { Parameters }\end{array}$} \\
\hline Age, years & $45 \pm 4.4$ & $\begin{array}{l}48.1 \pm \\
8.0\end{array}$ & $\begin{array}{l}47.8 \pm \\
7.6\end{array}$ & - & - & - & 0.139 & 1.000 & 1.000 \\
\hline Height, m & $\begin{array}{l}1.76 \pm \\
0.05\end{array}$ & $\begin{array}{l}1.74 \pm \\
0.09\end{array}$ & $\begin{array}{l}1.73 \pm \\
0.07\end{array}$ & - & - & - & 0.35 & 0.99 & 1.000 \\
\hline Weight, kg & $\begin{array}{l}93.5 \pm \\
14\end{array}$ & $\begin{array}{l}97.4 \pm \\
18\end{array}$ & $\begin{array}{l}99.1 \pm \\
25\end{array}$ & $\begin{array}{l}92.4 \pm \\
14\end{array}$ & $96.0 \pm 18$ & $\begin{array}{l}98.3 \pm \\
25\end{array}$ & 0.489 & 0.772 & 0.998 \\
\hline $\mathrm{BMI}, \mathrm{kg} / \mathrm{m}^{2}$ & $\begin{array}{l}30.1 \pm \\
3.7\end{array}$ & $\begin{array}{l}32.1 \pm \\
4.4\end{array}$ & $\begin{array}{l}32.5 \pm \\
6.3\end{array}$ & $\begin{array}{l}29.8 \pm \\
3.6\end{array}$ & $\begin{array}{l}31.7 \pm \\
4.4\end{array}$ & $\begin{array}{l}32.3 \pm \\
7.0\end{array}$ & 0.115 & 0.717 & 0.995 \\
\hline \multicolumn{10}{|l|}{$\begin{array}{l}\text { Hemodynamic } \\
\text { Parameters }\end{array}$} \\
\hline $\mathrm{HR}$ resting, $\mathrm{bpm}$ & $69 \pm 4.9$ & $74 \pm 7.5^{a}$ & $76 \pm 9.2^{a}$ & $\begin{array}{l}66 \pm \\
6.4^{a}\end{array}$ & $69 \pm 7.9^{b}$ & $\begin{array}{l}71 \pm \\
8.6^{c}\end{array}$ & 0.011 & 0.004 & 0.687 \\
\hline SBP, mmHg & $\begin{array}{l}140 \pm \\
7.3\end{array}$ & $\begin{array}{l}123 \pm \\
16^{a}\end{array}$ & $\begin{array}{l}121 \pm \\
12^{a}\end{array}$ & $\begin{array}{l}115 \pm \\
13^{a}\end{array}$ & $112 \pm 14^{b}$ & $\begin{array}{l}113 \pm \\
9.4^{c}\end{array}$ & 0.002 & $<0.001$ & 0.011 \\
\hline DBP, mmHg & $96 \pm 5.0$ & $82 \pm 11^{a}$ & $80 \pm 6.7^{a}$ & $\begin{array}{l}76 \pm \\
9.7^{a}\end{array}$ & $74 \pm 9.5^{b}$ & $\begin{array}{l}74 \pm \\
5.6^{c}\end{array}$ & $<0.001$ & $<0.001$ & 0.002 \\
\hline MBP, mmHg & $\begin{array}{l}114 \pm \\
4.2\end{array}$ & $99 \pm 12^{a}$ & $96 \pm 8.1^{a}$ & $89 \pm 10^{a}$ & $89 \pm 11^{b}$ & $\begin{array}{l}90 \pm \\
6.2^{c}\end{array}$ & $<0.001$ & $<0.001$ & $<0.001$ \\
\hline \multicolumn{10}{|l|}{$\begin{array}{l}\text { Metabolic } \\
\text { Parameters }\end{array}$} \\
\hline $\mathrm{VO}_{2 \text { peak }}, \mathrm{mL} / \mathrm{min} / \mathrm{kg}$ & $\begin{array}{l}23.9 \pm \\
5.3\end{array}$ & $\begin{array}{l}23.4 \pm \\
4.3\end{array}$ & $\begin{array}{l}21.9 \pm \\
4.0\end{array}$ & $\begin{array}{l}31.3 \pm \\
6.5^{a}\end{array}$ & $\begin{array}{l}30.22 \pm \\
6.3^{b}\end{array}$ & $\begin{array}{l}28.5 \pm \\
3.9^{c}\end{array}$ & 0.155 & $<0.001$ & 0.958 \\
\hline $\begin{array}{l}\text { Total Cholesterol, } \\
\mathrm{mg} / \mathrm{dL}\end{array}$ & $222 \pm 38$ & $206 \pm 42$ & $203 \pm 30$ & $\begin{array}{l}200 \pm \\
24^{a}\end{array}$ & $194 \pm 41^{b}$ & $\begin{array}{l}195 \pm \\
29^{c}\end{array}$ & 0.315 & 0.04 & 0.68 \\
\hline $\mathrm{HDL}, \mathrm{mg} / \mathrm{dL}$ & $\begin{array}{l}45.8 \pm \\
9.5\end{array}$ & $\begin{array}{l}40.3 \pm \\
8.3\end{array}$ & $\begin{array}{l}39.2 \pm \\
8.7\end{array}$ & $\begin{array}{l}46.3 \pm \\
6.5\end{array}$ & $43.1 \pm 11$ & $\begin{array}{l}43.7 \pm \\
8.1^{c}\end{array}$ & 0.06 & 0.14 & 0.66 \\
\hline $\mathrm{LDL}, \mathrm{mg} / \mathrm{dL}$ & $148 \pm 31$ & $133 \pm 34$ & $134 \pm 28$ & $\begin{array}{l}136 \pm \\
22^{a}\end{array}$ & $122 \pm 28^{b}$ & $138 \pm 32$ & 0.106 & 0.24 & 0.48 \\
\hline Triglycerides, mg/dL & $143 \pm 39$ & $\begin{array}{l}186 \pm \\
189\end{array}$ & $164 \pm 73$ & $\begin{array}{l}106 \pm \\
47^{a}\end{array}$ & $\begin{array}{l}166 \pm \\
176\end{array}$ & $156 \pm 60$ & 0.2 & 0.37 & 0.89 \\
\hline Glucose, mg/dL & $96 \pm 12$ & $112 \pm 35$ & $104 \pm 25$ & $96 \pm 11$ & $94 \pm 13^{b}$ & $99 \pm 23^{c}$ & 0.37 & 0.07 & 0.19 \\
\hline
\end{tabular}

Anthropometric characteristics, cardiorespiratory fitness, and blood parameters evaluated are also shown in Table 1. The results showed that there were no differences in anthropometric characteristics before and after aerobic physical training between the studied groups. The $\mathrm{VO}_{2 \text { peak }}$ showed similarities between the groups, even after the increase resulting from aerobic physical training. Regarding laboratory tests, the groups showed similar values for almost all parameters evaluated before and after aerobic physical training. In this case, only total cholesterol levels were significantly lower after aerobic physical training.

Figure 2 and Table 2 show the parameters of HRV obtained in the supine position, before and after aerobic physical training. The results show that the losartan group had lower values of HF oscillation in absolute units compared to the control group. After 16 weeks of aerobic physical training, the control and enalapril groups showed increases in total variance and in LF and HF oscillations in absolute units, while the losartan group only showed increases in total variance and HF oscillations in absolute units. 
Table 2

Values of heart rate variability, systolic blood pressure variability, and spontaneous baroreflex sensitivity obtained in all groups, before and after the 16 -week aerobic physical training.

\begin{tabular}{|c|c|c|c|c|c|c|c|c|c|}
\hline & \multicolumn{3}{|c|}{ Before training } & \multicolumn{3}{|c|}{ After training } & \multirow{2}{*}{$\begin{array}{l}\text { Drug } \\
\text { Factor } \\
\mathrm{P}\end{array}$} & \multirow{2}{*}{$\begin{array}{l}\text { Training } \\
\text { Factor }\end{array}$} & \multirow{2}{*}{$\begin{array}{l}\text { Interaction } \\
\mathrm{P}\end{array}$} \\
\hline & Control & Losartan & Enalapril & Control & Losartan & Enalapril & & & \\
\hline \multicolumn{10}{|l|}{ HR Variability } \\
\hline Variance, $\mathrm{ms}^{2}$ & $\begin{array}{l}1106 \pm \\
380\end{array}$ & $\begin{array}{l}847 \pm \\
534\end{array}$ & $\begin{array}{l}824 \pm \\
581\end{array}$ & $\begin{array}{l}1330 \pm \\
617\end{array}$ & $\begin{array}{l}1349 \pm \\
679^{b}\end{array}$ & $\begin{array}{l}1434 \pm \\
1097^{c}\end{array}$ & 0.75 & 0.001 & 0.5 \\
\hline $\mathrm{LF}, \mathrm{ms}^{2}$ & $\begin{array}{l}329 \pm \\
152\end{array}$ & $\begin{array}{l}306 \pm \\
221\end{array}$ & $\begin{array}{l}267 \pm \\
211\end{array}$ & $\begin{array}{l}422 \pm \\
250\end{array}$ & $\begin{array}{l}356 \pm \\
212\end{array}$ & $\begin{array}{l}534 \pm \\
329^{c}\end{array}$ & 0.7 & 0.017 & 0.42 \\
\hline LF, nu & $\begin{array}{l}60 \pm \\
9.8\end{array}$ & $67 \pm 17$ & $63 \pm 19$ & $57 \pm 17$ & $62 \pm 17^{b}$ & $58 \pm 12$ & 0.26 & 0.14 & 0.96 \\
\hline $\mathrm{HF}, \mathrm{ms}^{2}$ & $\begin{array}{l}225 \pm \\
151\end{array}$ & $\begin{array}{l}136 \pm \\
101^{a}\end{array}$ & $\begin{array}{l}174 \pm \\
154\end{array}$ & $\begin{array}{l}309 \pm \\
199^{a}\end{array}$ & $\begin{array}{l}230 \pm \\
164^{b}\end{array}$ & $\begin{array}{l}447 \pm \\
357^{c}\end{array}$ & 0.041 & 0.001 & 0.32 \\
\hline $\mathrm{HF}, \mathrm{nu}$ & $\begin{array}{l}40 \pm \\
9.8\end{array}$ & $33 \pm 17$ & $37 \pm 19$ & $43 \pm 17$ & $38 \pm 17^{b}$ & $42 \pm 12$ & 0.26 & 0.14 & 0.96 \\
\hline Ratio LF/HF & $\begin{array}{l}1.65 \pm \\
0.6\end{array}$ & $\begin{array}{l}3.16 \pm \\
2.6^{a}\end{array}$ & $\begin{array}{l}2.44 \pm \\
1.8\end{array}$ & $\begin{array}{l}1.8 \pm \\
1.4\end{array}$ & $\begin{array}{l}2.47 \pm \\
2.4\end{array}$ & $\begin{array}{l}1.75 \pm \\
1.3\end{array}$ & 0.049 & 0.27 & 0.58 \\
\hline \multicolumn{10}{|l|}{$\begin{array}{l}\text { SBP } \\
\text { Variability }\end{array}$} \\
\hline $\begin{array}{l}\text { Variance, } \\
\mathrm{mmHg}^{2}\end{array}$ & $\begin{array}{l}24.5 \pm \\
8.3\end{array}$ & $22 \pm 8.2$ & $27 \pm 10$ & $25 \pm 11$ & $21 \pm 7.4$ & $30 \pm 9.5$ & 0.004 & 0.66 & 0.58 \\
\hline $\mathrm{LF}, \mathrm{mmHg}^{2}$ & $\begin{array}{l}8.8 \pm \\
3.8\end{array}$ & $7.3 \pm 3.3$ & $6.9 \pm 2.8$ & $\begin{array}{l}6.7 \pm \\
2.7^{a}\end{array}$ & $6.7 \pm 3.5$ & $8.2 \pm 3.5$ & 0.59 & 0.47 & 0.12 \\
\hline \multicolumn{10}{|l|}{$\begin{array}{l}\text { Spontaneous } \\
\text { BRS }\end{array}$} \\
\hline BEI & $\begin{array}{l}0.55 \pm \\
0.13\end{array}$ & $\begin{array}{l}0.45 \pm \\
0.18\end{array}$ & $\begin{array}{l}0.48 \pm \\
0.18\end{array}$ & $\begin{array}{l}0.64 \pm \\
0.11^{a}\end{array}$ & $\begin{array}{l}0.48 \pm \\
0.17\end{array}$ & $\begin{array}{l}0.49 \pm \\
0.18\end{array}$ & 0.002 & 0.19 & 0.61 \\
\hline Ramps $n^{\circ}$ & $86 \pm 37$ & $70 \pm 43$ & $82 \pm 37$ & $96 \pm 47$ & $61 \pm 39$ & $76 \pm 30$ & 0.025 & 0.79 & 0.54 \\
\hline $\begin{array}{l}\text { Gain, } \\
\mathrm{ms} / \mathrm{mmHg}\end{array}$ & $\begin{array}{l}6.7 \pm \\
3.3\end{array}$ & $\begin{array}{l}6.43 \pm \\
2.6\end{array}$ & $7.0 \pm 5.3$ & $\begin{array}{l}8.46 \pm \\
4.6^{a}\end{array}$ & $\begin{array}{l}8.4 \pm \\
3.0^{b}\end{array}$ & $\begin{array}{l}9.27 \pm \\
5.4^{c}\end{array}$ & 0.73 & 0.013 & 0.97 \\
\hline $\begin{array}{l}\text { Gain down, } \\
\mathrm{ms} / \mathrm{mmHg}\end{array}$ & $\begin{array}{l}6.95 \pm \\
3.3\end{array}$ & $\begin{array}{l}6.57 \pm \\
2.5\end{array}$ & $7.2 \pm 5.5$ & $\begin{array}{l}9.05 \pm \\
5.1^{a}\end{array}$ & $\begin{array}{l}8.52 \pm \\
2.9^{b}\end{array}$ & $\begin{array}{l}9.0^{ \pm} \\
5.4^{c}\end{array}$ & 0.83 & 0.02 & 0.99 \\
\hline $\begin{array}{l}\text { Gain up, } \\
\mathrm{ms} / \mathrm{mmHg}\end{array}$ & $\begin{array}{l}6.31 \pm \\
3.3\end{array}$ & $\begin{array}{l}6.34 \pm \\
2.8\end{array}$ & $\begin{array}{l}6.83 \pm \\
4.7\end{array}$ & $\begin{array}{l}7.96 \pm \\
4.2^{a}\end{array}$ & $\begin{array}{l}8.29 \pm \\
3.1^{b}\end{array}$ & $\begin{array}{l}9.41 \pm \\
5.5^{c}\end{array}$ & 0.56 & 0.009 & 0.88 \\
\hline
\end{tabular}

Figure 2 and Table 3 show the HRV parameters obtained after the tilt test, before and after aerobic physical training. The control and enalapril groups showed an increase in LF oscillations in normalized units and a reduction in HF oscillations in absolute and normalized units compared with their respective groups in the supine position. However, no changes were observed in the losartan group. After aerobic physical training, the control group showed an increase in LF oscillations in normalized units and a reduction in HF oscillations in absolute and normalized units. In turn, the losartan group showed only a decrease in the total variance, while the enalapril group showed a reduction in the HF oscillations in absolute units compared with the results obtained in the supine position. The intergroup comparison of the spectral parameters obtained after orthostatism before aerobic physical training showed that the enalapril group showed a reduction in total variance compared to the control group. It also showed a decrease in HF oscillations in the normalized unit compared to that in the losartan group. In turn, after physical training, we observed that the control group showed an increase in total variance and the losartan group showed an increase in $\mathrm{HF}$ oscillations in absolute units, both compared with their respective untrained group, while the enalapril group showed increases in total variance and HF oscillations in absolute and normalized units, in addition to the reduction in LF oscillations in normalized units. We also observed that the trained enalapril group showed an increase in HF oscillations in the normalized unit compared with the trained control group. 
Table 3

Values of heart rate variability, systolic blood pressure variability, and spontaneous baroreflex sensitivity obtained after tilt test, before and after the 16 -week physical training.

\begin{tabular}{|c|c|c|c|c|c|c|c|c|c|}
\hline & \multicolumn{3}{|c|}{ Before training } & \multicolumn{3}{|c|}{ After training } & \multirow{2}{*}{$\begin{array}{l}\begin{array}{l}\text { Drug } \\
\text { Factor }\end{array} \\
\mathrm{P}\end{array}$} & $\begin{array}{l}\text { Training } \\
\text { Factor }\end{array}$ & \multirow[t]{2}{*}{ Int } \\
\hline & Control & Losartan & Enalapril & Control & Losartan & Enalapril & & $P$ & \\
\hline \multicolumn{10}{|l|}{$\begin{array}{l}\text { HR } \\
\text { Variability }\end{array}$} \\
\hline $\begin{array}{l}\text { Variance, } \\
\mathrm{ms}^{2}\end{array}$ & $\begin{array}{l}1054 \pm \\
616\end{array}$ & $\begin{array}{l}804 \pm \\
422\end{array}$ & $\begin{array}{l}587 \pm \\
520\end{array}$ & $\begin{array}{l}1212 \pm \\
707^{a}\end{array}$ & $\begin{array}{l}978 \pm \\
625\end{array}$ & $\begin{array}{l}1104 \pm \\
724^{c}\end{array}$ & 0.034 & 0.003 & 0.26 \\
\hline $\mathrm{LF}, \mathrm{ms}^{2}$ & $\begin{array}{l}392 \pm \\
256\end{array}$ & $\begin{array}{l}277 \pm \\
166\end{array}$ & $\begin{array}{l}222 \pm \\
190\end{array}$ & $\begin{array}{l}342 \pm \\
244^{a}\end{array}$ & $\begin{array}{l}331 \pm \\
212\end{array}$ & $\begin{array}{l}366 \pm \\
217^{c}\end{array}$ & 0.33 & 0.24 & 0.19 \\
\hline LF, nu & $\begin{array}{l}81 \pm \\
9.2\end{array}$ & $70 \pm 19$ & $79 \pm 12$ & $\begin{array}{l}76 \pm \\
13\end{array}$ & $69 \pm 18$ & $65 \pm 16^{c}$ & 0.045 & 0.031 & 0.23 \\
\hline $\mathrm{HF}, \mathrm{ms}^{2}$ & $\begin{array}{l}94 \pm \\
96\end{array}$ & $111 \pm 85$ & $63 \pm 83$ & $\begin{array}{l}128 \pm \\
164\end{array}$ & $\begin{array}{l}185 \pm \\
253\end{array}$ & $\begin{array}{l}212 \pm \\
168^{c}\end{array}$ & 0.59 & 0.006 & 0.32 \\
\hline HF, nu & $\begin{array}{l}19 \pm \\
9.2\end{array}$ & $30 \pm 19$ & $21 \pm 12$ & $\begin{array}{l}24 \pm \\
13\end{array}$ & $31 \pm 18$ & $35 \pm 16^{c}$ & 0.045 & 0.031 & 0.23 \\
\hline Ratio LF/HF & $\begin{array}{l}5.14 \pm \\
2.2\end{array}$ & $\begin{array}{l}5.26 \pm \\
6.3\end{array}$ & $\begin{array}{l}7.76 \pm \\
13\end{array}$ & $\begin{array}{l}4.35 \pm \\
2.7\end{array}$ & $\begin{array}{l}4.11 \pm \\
4.9\end{array}$ & $3.6 \pm 5.4$ & 0.79 & 0.12 & 0.53 \\
\hline \multicolumn{10}{|l|}{$\begin{array}{l}\text { SBP } \\
\text { Variability }\end{array}$} \\
\hline $\begin{array}{l}\text { Variance, } \\
\mathrm{mmHg}^{2}\end{array}$ & $\begin{array}{l}47 \pm \\
23\end{array}$ & $34 \pm 19$ & $33 \pm 15$ & $\begin{array}{l}35 \pm \\
16\end{array}$ & $27 \pm 15^{b}$ & $32 \pm 14$ & 0.029 & 0.059 & 0.44 \\
\hline $\mathrm{LF} \mathrm{mmHg}{ }^{2}$ & $\begin{array}{l}21 \pm \\
14\end{array}$ & $17 \pm 10$ & $14 \pm 10$ & $\begin{array}{l}16 \pm \\
11\end{array}$ & $14 \pm 10$ & $12 \pm 8.5$ & 0.19 & 0.13 & 0.85 \\
\hline \multicolumn{10}{|c|}{$\begin{array}{l}\text { Spontaneous } \\
\text { BRS }\end{array}$} \\
\hline BEI & $\begin{array}{l}0.44 \pm \\
0.14\end{array}$ & $\begin{array}{l}0.39 \pm \\
0.19\end{array}$ & $\begin{array}{l}0.31 \pm \\
0.19\end{array}$ & $\begin{array}{l}0.5 \pm \\
0.19\end{array}$ & $\begin{array}{l}0.41 \pm \\
0.18\end{array}$ & $\begin{array}{l}0.36 \pm \\
0.17^{c}\end{array}$ & 0.015 & 0.23 & 0.9 \\
\hline Ramps $n^{\circ}$ & $\begin{array}{l}81 \pm \\
34\end{array}$ & $65 \pm 33$ & $56 \pm 39$ & $\begin{array}{l}92 \pm \\
34\end{array}$ & $63 \pm 37^{d}$ & $64 \pm 38^{c}$ & 0.006 & 0.42 & 0.66 \\
\hline $\begin{array}{l}\text { Gain, } \\
\mathrm{ms} / \mathrm{mmHg}\end{array}$ & $\begin{array}{l}4.67 \pm \\
2.0\end{array}$ & $5.6 \pm 2.8$ & $\begin{array}{l}4.72 \pm \\
3.0\end{array}$ & $\begin{array}{l}5.69 \pm \\
3.4\end{array}$ & $\begin{array}{l}5.72 \pm \\
2.2\end{array}$ & $\begin{array}{l}6.17 \pm \\
3.5^{c}\end{array}$ & 0.77 & 0.12 & 0.58 \\
\hline $\begin{array}{l}\text { Gain down, } \\
\mathrm{ms} / \mathrm{mmHg}\end{array}$ & $\begin{array}{l}4.98 \pm \\
2.1\end{array}$ & $\begin{array}{l}5.33 \pm \\
2.2\end{array}$ & $\begin{array}{l}4.84 \pm \\
2.8\end{array}$ & $\begin{array}{l}6.23 \pm \\
3.7^{a}\end{array}$ & $\begin{array}{l}6.03 \pm \\
2.2\end{array}$ & $\begin{array}{l}6.03 \pm \\
3.5^{c}\end{array}$ & 0.93 & 0.057 & 0.89 \\
\hline $\begin{array}{l}\text { Gain up, } \\
\mathrm{ms} / \mathrm{mmHg}\end{array}$ & $\begin{array}{l}4.21 \pm \\
1.9\end{array}$ & $\begin{array}{l}5.76 \pm \\
3.6\end{array}$ & $\begin{array}{l}4.55 \pm \\
3.2\end{array}$ & $\begin{array}{l}4.9 \pm \\
3.4\end{array}$ & $\begin{array}{l}5.38 \pm \\
2.4\end{array}$ & $\begin{array}{l}6.29 \pm \\
3.6^{c}\end{array}$ & 0.35 & 0.26 & 0.34 \\
\hline
\end{tabular}

Values expressed as means \pm SD. HR, heart rate; LF, low frequency; HF, high frequency; ms, milliseconds, nu, normalized units; SBP, systolic blood pressure; $m$ millimetres of mercury; BRS, baroreflex sensitivity; BEI, baroreflex effectiveness index. ${ }^{a} \mathrm{P}<0.05$ vs. Control before training; ${ }^{b}$ Losartan before training; ${ }^{c}$ Enala before training.

Table 2 shows the autonomic parameters of BPV and spontaneous BRS in all groups, obtained in the supine position, before and after aerobic physical training. BPV results showed intergroup differences after aerobic physical training, and the enalapril group showed an increase in variance compared to the losartan group. In turn, the BRS results showed that all groups had similar responses before aerobic physical training. After 16 weeks of aerobic physical training, all groups showed an increase in BRS, characterized by an increase in the total gain for bradycardic (Up) and tachycardic (Down) responses in relation to their respective values obtained before aerobic physical training. In addition, the losartan group showed a reduction in the BEI, and number of ramps compared to the control group, while the enalapril group showed a reduction only in the BEl compared to the control group.

Table 3 also shows the BPV and BRS parameters in all groups, but obtained during the tilt test, and before and after aerobic physical training. BPV results showed intergroup differences before aerobic physical training, in which the enalapril group showed a decrease in variance compared to the control group. In turn, the BRS results showed that the enalapril group had a reduction in BEl and ramp numbers in relation to the control group, while the losartan group showed a reduction only in ramp numbers when compared with the control group. After aerobic physical training, the number of ramps remained reduced in the losartan and enalapril groups compared with the control group. In turn, during the tilt test, no differences were observed before and after aerobic physical training in any of the BRS parameters.

\section{Discussion}


The HRV indices and BRS gain reflect the cardiovascular system's ability to reorganize itself due to variations in the demands of the internal environment. These autonomic parameters are recognized as important predictors of morbidity and mortality [19-21]. In this study, the results indicated that aerobic physical training is a therapeutic tool that plays a fundamental role to positively regulates the cardiovascular autonomic control in hypertensive patients regardless of pharmacological treatment.

Long-term treatment with losartan and enalapril was not accompanied by improved cardiovascular autonomic control, although BP was maintained at values considered normal. In fact, when compared with untreated hypertensive volunteers, the results suggest that pharmacological treatments with reninangiotensin system (RAS) inhibitors in the long-term seem to promote a reduction in the autonomic modulation of HRV, attenuating the variation intensity of each autonomic component, including during the tilt test. Some parameters, such as the HF oscillations of HRV, in the losartan group had lower values than the untreated hypertensive group [7, 8]. Moreover, when this group was subjected to a tilt test, the autonomic parameters evaluated did not differ from those in supine position in absolute units, which is not common. This suggests that in addition to the absence of positive cardiovascular autonomic effects, treatment with losartan appears to decrease the autonomic regulatory efficacy. Likewise, treatment with enalapril alone did not result in major autonomic benefits, but it appears that enalapril has some advantages over losartan. The reasons for these observations are uncertain. However, it is important to highlight the mechanisms of action of these two antihypertensive drugs. While enalapril reduces the formation of angiotensin II by inhibiting the angiotensin-converting enzyme (ACE), it also contributes to the reduction of bradykinin degradation. The greater bioavailability of bradykinin promotes greater formation of nitric oxide (NO) and vasodilating prostaglandins, further reducing the BP, and promoting its effects on the vascular beds, heart, and kidneys [30-32]. However, this increase in bradykinin concentration also seems to be responsible for a greater activation of the cardiac sympathetic afferent reflex, resulting in the attenuation of cardiovascular autonomic modulation [33]. In turn, losartan has been pointed out in the literature as being related to an important factor that interferes with the trophic effect of angiotensin II on sympathetic autonomic modulation [34-37].

Thus, blocking $\mathrm{AT}_{1}$ receptors appears to result in cardiac autonomic effects that are slightly different from that of enalapril. An experimental study with spontaneously hypertensive rats (SHRs) treated with losartan or enalapril maleate showed that both had reduced BP but did not promote any change in cardiac autonomic modulation. Regarding BRS, only enalapril group showed an increase in gain. When these animals were trained, a better cardiac autonomic response was observed, and the association of aerobic physical training with enalapril resulted in greater gains. The authors pointed out that these differences would involve two aspects: first is related to a greater decrease in angiotensin II levels, and second to the improvement of endothelial function. In the first case, the aerobic training effects was to interfere with the pathways of Elastase-2 and chymase, which are alternative forms of angiotensin II production that do not depend on ACE [26]. This action would further decrease the concentration of angiotensin II, including the central nervous system, potentiating the positive effects of enalapril. Other studies have shown an increase in Elastase-2 in the arteries, lungs, and heart of SHRs [38, 39], as well as a higher concentration of mRNA levels for chymase in these animals compared to normotensive rats [40]. In addition, in vascular and cardiac pathways, elastase-2 and chymase become potentially activated in SHRs treated with enalapril maleate due to ACE inhibition [39, 41]. However, new approaches are needed to elucidate the interaction between aerobic physical training effects and these mechanisms. The second aspect is related to a relationship with endothelial function recovery, which responds with greater production of $\mathrm{NO}$ and other dilating factors. This can be caused by the reduction of angiotensin II, as well as by the increase in bradykinin concentration, both of which results from aerobic physical training, through greater vascular shear stress [42-44]. In this condition, NO seems to have an effective action in reducing sympathetic participation and increasing BRS [45, 46].

In fact, our study reinforces the importance of aerobic physical training as a fundamental therapy for the treatment of hypertensive patients. We know that its therapeutic effects are broad and systemic $[12,13,47]$. More specifically, on the cardiovascular system, the benefits related to cardiac morphological and functional adaptations are relevant, making the heart more efficient and less dependent on sympathetic autonomic influence, especially during rest and activities of daily living $[11,16,17]$. Gains on BRS are also observed, which is the result of adaptations in the autonomic reflex arc involving even the central neural nuclei of cardiovascular control, resulting in a more efficient cardiac regulation, characterized by a more balanced autonomic rearrangement. This autonomic rearrangement can be seen through increases in HRV and BRS, resulting in a greater adaptive capacity during physiological demands [48-51]. This new autonomic status induced by physical training can contribute to a more favourable positive outcome, not only in hypertensive patients, but also in patients with different conditions, mainly cardiovascular, metabolic, and immunological.

For all these benefits, the association of aerobic physical training with RAS inhibitors, mainly with enalapril, proved to be more advantageous for the control of hypertension and cardiac autonomic regulation. Regarding the differences found between enalapril and losartan before and after aerobic physical training, it is important to point out that this is a relevant finding and needs to be investigated, especially the observations obtained during the tilt test. The 'freezing' in HRV values of the spectral parameters observed in losartan group was surprising and needs to be explained, as it represents the main difference between the treatment with losartan and enalapril.

Thus, our findings confirm the aerobic physical training as an important tool that must be used in the hypertensive patients before and during pharmacological treatment. In this case, the association with pharmacological treatment is fundamental when aiming beyond BP reduction and gains in cardiovascular autonomic regulation. Furthermore, the cause of the differences found in cardiovascular autonomic regulation between losartan and enalapril are uncertain and need to be investigated.

\section{Abbreviations}

ACE - angiotensin-converting enzyme

ACEi - angiotensin-converting enzyme inhibitors

$\mathrm{AT}_{1}$ - type 1 angiotensin II receptor 
BEI - baroreflex effectiveness index

BMI- body mass index

$\mathrm{BP}$ - blood pressure

BPV - blood pressure variability

BRS - baroreflex sensitivity

ECG - electrocardiogram

HR - heart rate

HRV - heart rate variability

NO - nitric oxide

PI - pulse interval

RAS - renin-angiotensin system

RASi - renin-angiotensin system inhibitors

RRi - RR interval of electrocardiogram

SAH - systemic arterial hypertension

SBP - systolic blood pressure

SHRs - spontaneously hypertensive rats

$\mathrm{VCO}_{2}$ - carbon dioxide exhalation

$\mathrm{VO}_{2}$ - oxygen consumption

\section{Declarations}

Acknowledgements: The author expresses their sincere gratitude to all volunteers who participated in this study.

Source of Funding: This study was supported bygrant2018/08569-6, São Paulo Research Foundation (FAPESP).

Conflict of Interest: Tábata de Paula Facioli, Stella Vieira Philbois, Jen Tank, and Hugo Celso Dutra de Souza declare that they have no competing interests.

Availability of data and materials: The datasets used and/or analysed during the current study are available from the corresponding author on reasonable request.

Ethics approval and consent to participate: This study was approved by the Ethics Committee for Human Research from the "Hospital das Clinicas" (Medical School of Ribeirão Preto, University of São Paulo, Ribeirão Preto, Brazil) (Protocol number: 2.280.910/2017). All the procedures in this study were in accordance with the guidelines stated in the Declaration of Helsinki and Good Clinical Practice. Informed consent was obtained from all participants included in the study.

Consent for publication: Not applicable.

Author Contributions: TPF, SVP and HCDS contributed to the conception and design of the work. TPF and SVP contributed to the acquisition, analysis and interpretation of data and drafted the manuscript. TPF, SVP, JT and HCDS critically revised the manuscript for important intellectual content. All authors read and approved the final manuscript.

\section{References}

1. Weinstock M, Gorodetsky E. Comparison of the effects of angiotensin II, Losartan, and Enalapril on baroreflex control of heart rate in conscious rabbits. J Cardiovasc Pharmacol. 2005;25(3):501-7. https://doi.org/10.1097/00005344-199503000-00024

2. Guasti L, Petrozzino MR, Mainardi LT, Grimoldi P, Zanotta D, Garganico D, et al. Autonomic function and baroreflex sensitivity during angiotensinconverting enzyme inhibition or angiotensin II AT-1 receptor blockade in essential hypertensive patients. Acta Cardiol. 2001;56(5):289-

95. https://doi.org/10.2143/AC.56.5.2005689

3. Karas M, Lacourcière Y, Leblanc AR, Nadeau R, Dubé B, Florescu M, et al. Effect of the renin-angiotensin system or calcium channel blockade on the circadian variation of heart rate variability, blood pressure and circulating catecholamines in hypertensive patients. J Hypertens. 2005;23(6):125160. https://doi.org/10.1097/01.hjh.0000170389.69202.53 
4. Fu Q, Shook RP, Okazaki K, Hastings JL, Shibata S, Conner CL, et al. Vasomotor sympathetic neural control is maintained during sustained upright posture in humans. J Physiol. 2006;577(Pt 2):679-87. https://doi.org/10.1113/jphysiol.2006.118158

5. Lewandowski J, Abramczyk P, Dobosiewicz A, Bidiuk J, Sinski M, Gaciong Z. The effect of Enalapril and telmisartan on clinical and biochemical indices of sympathetic activity in hypertensive patients. Clin Exp Hypertens. 2008;30(5):423-32. https://doi.org/10.1080/10641960802279132

6. Milovanovic B, Trifunovic D, Djuric D. Autonomic nervous system adjustment (ANSA) in patients with hypertension treated with Enalapril. Acta Physiol Hung. 2011;98(1):71-84. https://doi.org/10.1556/APhysiol.98.2011.1.9

7. Heusser K, Vitkovsky J, Schmieder RE, Schobel HP. AT1 antagonism by eprosartan lowers heart rate variability and baroreflex gain. Auton Neurosci. 2003;107(1):45-51.

8. Heusser K, Vitkovsky J, Raasch W, Schmieder RE, Schobel HP. Elevation of sympathetic activity by eprosartan in young male subjects. Am J Hypertens. 2003;16(8):658-64. https://doi.org/10.1016/S0895-7061(03)00917-8

9. A report of the American College of Cardiology/American Heart Association Task Force on Clinical Practice Guidelines. 2017 Guideline for the Prevention, Detection, Evaluation, and Management of High Blood Pressure in Adults. J Am Coll Cardiol. 2017;23976. https://doi.org/10.1016/j.jacc.2017.07.745

10. The Task Force for the management of arterial hypertension of the European Society of Cardiology and the European Society of Hypertension. 2018 ESC/ESH Guidelines for the management of arterial hypertension. European Heart Journal. 2018;00:1-98. https://doi.org/10.1093/eurheartj/ehy339

11. Carter JB, Banister EW, Blaber AP. Effect of endurance exercise on autonomic control of heart rate. Sports Med. 2003;33(1):3346. https://doi.org/10.2165/00007256-200333010-00003

12. Cornelissen VA, Fagard RH. Effects of endurance training on blood pressure, blood pressure-regulating mechanismis, and cardiovascular risk factors. Hypertension. 2005;46(4):667-75. https://doi.org/10.1161/01.HYP.0000184225.05629.51

13. Laterza MC, De Matos L, Trombetta Cl, Braga AMW, Roveda F, Alves MJNN, et al. Exercise Training Restores Baroreflex Sensitivity in Never-Treated Hypertensive. Hypertension. 2007;49(6):1298-306. https://doi.org/10.1161/HYPERTENSIONAHA.106.085548

14. Piotrowicz E, Baranowski R, Piotrowska M, Zielinski T, Piotrowicz. Variable effects of physical training of heart rate variability, heart rate recovery, and heart rate turbulence in chronic heart failure. Pacing Clin Electrophysiol. 2009;32(suppl1):S113-15. https://doi.org/10.1111/j.1540-8159.2008.02266.X

15. Sloan RP, Shapiro PA, Demeersman RE, Bagiella E, Brondolo EN, McKinley PS, et al. The effect of aerobic training and cardiac autonomic regulation in young adults. Am J Public Health. 2009;99(5) 921-28. https://doi.org/10.2105/AJPH.2007.133165

16. Collier SR, Kanaley JA, Carhart Jr, R et al. Cardiac autonomic function and baroreflex changes following 4 weeks of resistance versus aerobic training in individuals with pre-hypertension. Acta Physiol (Oxf). 2009;195(3):339-48. https://doi.org/10.1111/j.1748-1716.2008.01897.x

17. Cozza IC, Di Sacco THR, Mazon JH, Salgado MCO, Dutra SGV, Cesarino EJ, et al. Physical exercise improves cardiac autonomic modulation in hypertensive patients independently of angiotensin-converting enzyme inhibitor treatment. Hypertens Res. 2012;35(1):82-7.

https://doi.org/10.1038/hr.2011.162

18. Souza HD, De Araujo JE, Martins-Pinge MC, Cozza IC, Martins-Dias DP. Nitric oxide synthesis blockade reduced the baroreflex sensitivity in trained rats. Auton Neurosci Basic Clin. 2009;150(1-2):38-44. https://doi.org/10.1016/j.autneu.2009.04.007

19. Doğru MT, Simşek V, Sahin O, Ozer N. Differences in autonomic activity in individuals with optimal, normal, and high-normal blood pressure levels. Turk Kardiyol Dern Ars. 2010;38(3):182-88.

20. Čelovská D, Stasko J, Gonsorčík J, Gašpar L, Štvrtinová V. The value of baroreflex sensitivity for cardiovascular risk stratification in hypertensives. Vnitr Lek. 2010;56(6):607-12.

21. Okada YM, Galbreath M, Shibatae S, Jarvis SS, VanGundy TB, Meier RL, et al. Relationship between sympathetic baroreflex sensitivity and arterial stiffness in elderly men and women. Hypertension. 2012;59(1):98-104. https://doi.org/10.1161/HYPERTENSIONAHA.111.176560

22. Dutra SGV, Pereira APM, Tezini GCSV, Mazon JH, Martins-Pinge MC, Souza HCD. Cardiac autonomic modulation is determined by gender and is independent of aerobic physical capacity in healthy subjects. PLoS ONE. 2013;8(10):e77092. https://doi.org/10.1371/journal.pone.0077092

23. Facioli TP, Gastaldi AC, Dutra SGV, Felix ACS, Philbois SV, Sánchez-Delgado JC, et al. The blood pressure variability and baroreflex sensitivity in healthy participants are not determined by sex or cardiorespiratory fitness. Blood Press Monit. 2018;23(5):260-70.

https://doi.org/10.1097/MBP.0000000000000338

24. Philbois SV, Facioli TP, Gastaldi AC, Rodrigues JAL, Tank J, Fares TH, Rodrigues KP, Souza HCD. Important differences between hypertensive middle-aged women and men in cardiovascular autonomic control-a critical appraisal. Biol Sex Differ. 2021;11;12(1):11. https://doi.org/10.1186/s13293-020-00355y.

25. Balke B, Ware RW. An experimental study of physical fitness of Air Force personnel. U S Armed Forces Med J. 1959;10(6):675-88.

26. Tezini GCSV, Dias DPM, Souza HCD. Aerobic physical training has little effect on cardiovascular autonomic control in aging rats subjected to early menopause. Exp Gerontol. 2013;48(2):147-53. https://doi.org/10.1016/j.exger.2012.11.009

27. Maida KD, Gastaldi AC, de Paula Facioli T, de Araújo JE, de Souza HCD. Physical training associated with Enalapril but not to Losartan, results in better cardiovascular autonomic effects. Auton Neurosci. 2017;203:33-40. https://doi.org/10.1016/j.autneu.2016.12.002

28. Smyth HS, Sleight P, Pickering GW. Reflex regulation of arterial pressure during sleep in man. A quantitative method of assessing baroreflex sensitivity. Circ Res. 1969;24(1):109-21. https://doi.org/10.1161/01.RES.24.1.109

29. Bristow JD, Honour AJ, Pickering GW, Sleight P, Smyth HS. Diminished baroreflex sensitivity in high blood pressure. Circulation. 1969;39(1):48-54. https://doi.org/10.1161/01.CIR.39.1.48

30. Cushman DW, Cheung HS, Sabo EF, Ondetti MA. Design of potent competitive inhibitors of angiotensin-converting enzyme. Carboxyalkanoyl and mercaptoalkanoyl amino acids. Biochemistry. 1977;16(25):5484-91. https://doi.org/10.1021/bi00644a014

Page $10 / 13$ 
31. Ferrari R, Fox K. Insight into the mode of action of ACE inhibition in coronary artery disease: the ultimate 'EUROPA' story. Drugs. 2009;69:265-77. https://doi.org/10.2165/00003495-200969030-00003

32. Vijayaraghavan K, Deedwania P. Renin-angiotensin-aldosterone blockade for cardiovascular disease prevention. Cardiol Clin. 2011;29(1):137-56. https://doi.org/10.1016/j.ccl.2010.11.003

33. Koba S, Xing J, Sinoway LI, Li J. Bradykinin receptor blockade reduces sympathetic nerve response to muscle contraction in rats with ischemic heart failure. Am J Physiol Heart Circ Physiol. 2010;298(5):H1438-44. https://doi.org/10.1152/ajpheart.00558.2009

34. Williams B. Drug discovery in renin-angiotensin system intervention: past and future. Ther Adv Cardiovasc Dis. 2016;10(3):11825. https://doi.org/10.1177/1753944716642680

35. Culman J, Von Heyer C, Piepenburg B, Rascher W, Unger T. Effects of systemic treatment with irbesartan and Losartan on central responses to angiotensin II in conscious, normotensive rats. Eur J Pharmacol. 1999;367(2-3):255-65. https://doi.org/10.1016/s0014-2999 (98) 00983-2

36. Peterson RC, Dunlap ME. Angiotensin II receptor blockers in the treatment of heart failure. Congest Heart Fail. 2002;8(5):24650;256. https://doi.org/10.1111/j.1527-5299.2000.01156.x

37. Inaba S, Iwai M, Furuno M, Kanno H, Senba I, Okayama H, et al. Temporary Treatment with AT1 Receptor Blocker, Valsartan, From Early Stage of Hypertension Prevented Vascular Remodeling. Am J Hypertens. 2011;24(5):550-6. https://doi.org/10.1038/ajh.2011.6

38. Santos CF, Caprio MAV, Oliveira EB, Salgado MCO, Schipper DN, Munzenmaier DH, et al. Functional role, cellular source, and tissue distribution of rat elastase-2, an angiotensin Il-forming enzyme. Am J Pysiol Heart Circ Physiol. 2003;285(2):H775-83. https://doi.org/10.1152/ajpheart.00818.2002

39. Becari C, Teixeira FR, Oliveira EB, Salgado MCO. Angiotensin-converting enzyme inhibition augments the expression of rat elastase-2, an angiotensin IIforming enzyme. Am J Physiol Heart Circ Physiol. 2011;301(2):H565-70. https://doi.org/10.1152/ajpheart.00534.2010

40. Kirimura K, Takai S, Jin D, Muramatu M, Kishi K, Yoshikawa K, et al. Role of chymase-dependent angiotensin II formation in regulating blood pressure in spontaneously hypertensive rats. Hypertens Res. 2005;28(5):457-64. https://doi.org/10.1291/hypres.28.457

41. Paula CA, Sousa MV, Salgado MC, Oliveira EB. Purification and substrate specificity of an angiotensin converting elastase-2 from the rat mesenteric arterial bed perfusate. Biochim Biophys Acta. 1998;1388(1):227-38. https://doi.org/10.1016/s0167-4838 (98) 00186-1

42. Shen W, Zhang X, Wolin MS, Sessa W, Hintze TH. Nitric oxide production and NO synthase gene expression contribute to vascular regulation during exercise. Med Sci Sports Exerc. 1995;27(8):1125-34.

43. Vanhoutte PM, Shimokawa H, Tang EHC, Feletou M. Endothelial dysfunction and vascular disease. Acta Physiol (Oxf). 2009;196(2):193222. https://doi.org/10.1111/j.1748-1716.2009.01964.x

44. Duncker DJ, Bache RJ. Regulation of coronary blood flow during exercise. Physiol Rev. 2008;88(3):1009-86. https://doi.org/10.1152/physrev.00045.2006.

45. Souza HC, Ballejo G, Salgado MC, Da Silva VJ, Salgado HC. Cardiac sympathetic overactivity and decreased baroreflex sensitivity in L-NAME hypertensive rats. Am J Physiol Heart Circ Physiol. 2001;280(2):H844-50. https://doi.org/10.1152/ajpheart.2001.280.2.H844

46. Elayan HH, Kennedy BP, Ziegler MG. L-NAME raises systolic blood pressure in the pithed rat by a direct adrenal epinephrine releasing action. Life Sciences. 2002;70(21):2481-91. https://doi.org/10.1016/s0024-3205 (02) 01520-5

47. Mizuno Y, Jacob RF, Mason RP. Effects of calcium channel and renin-angiotensin system blockade on intravascular and neurohormonal mechanisms of hypertensive vascular disease. Am J Hypertens. 2008;21(10):1076-85. https://doi.org/10.1038/ajh.2008.258

48. De Abreu SB, Lenhard A, Mehanna A, De Souza HCD, De Aguiar Correa FM, Hasser EM, Martins-Pinge MC. Role of paraventricular nucleus in exercise training-induced autonomic modulation in conscious rats. Auton Neurosci. 2009;148(1-2):28-35. https://doi.org/10.1016/j.autneu.2009.02.007

49. Mastelari RB, De Souza HC, Lenhard A, De Aguiar Corrêa FM, Martins-Pinge MC. Nitric oxide inhibition in paraventricular nucleus on cardiovascular and autonomic modulation after exercise training in unanesthetized rats. Brain Res. 2011;1375:68-76. https://doi.org/10.1016/j.brainres.2010.12.049

50. Barbosa-Neto O, Abate DTRS, Marocolo Júnior M, Mota GR, Orsatti FL, Rossi e Silva RC et al. Exercise training improves cardiovascular autonomic activity and attenuates renal damage in spontaneously hypertensive rats. J Sports Sci Med. 2013;12(1):52-59.

51. Masson GS, Nair AR, Silva Soares PP, Michelini LC, Francis J. Aerobic training normalizes autonomic dysfunction, HMGB1 content, microglia activation and inflammation in hypothalamic paraventricular nucleus of SHR. Am J Physiol Heart Circ Physiol. 2015;309(7):H1115-22.

https://doi.org/10.1152/ajpheart.00349.2015

\section{Figures}




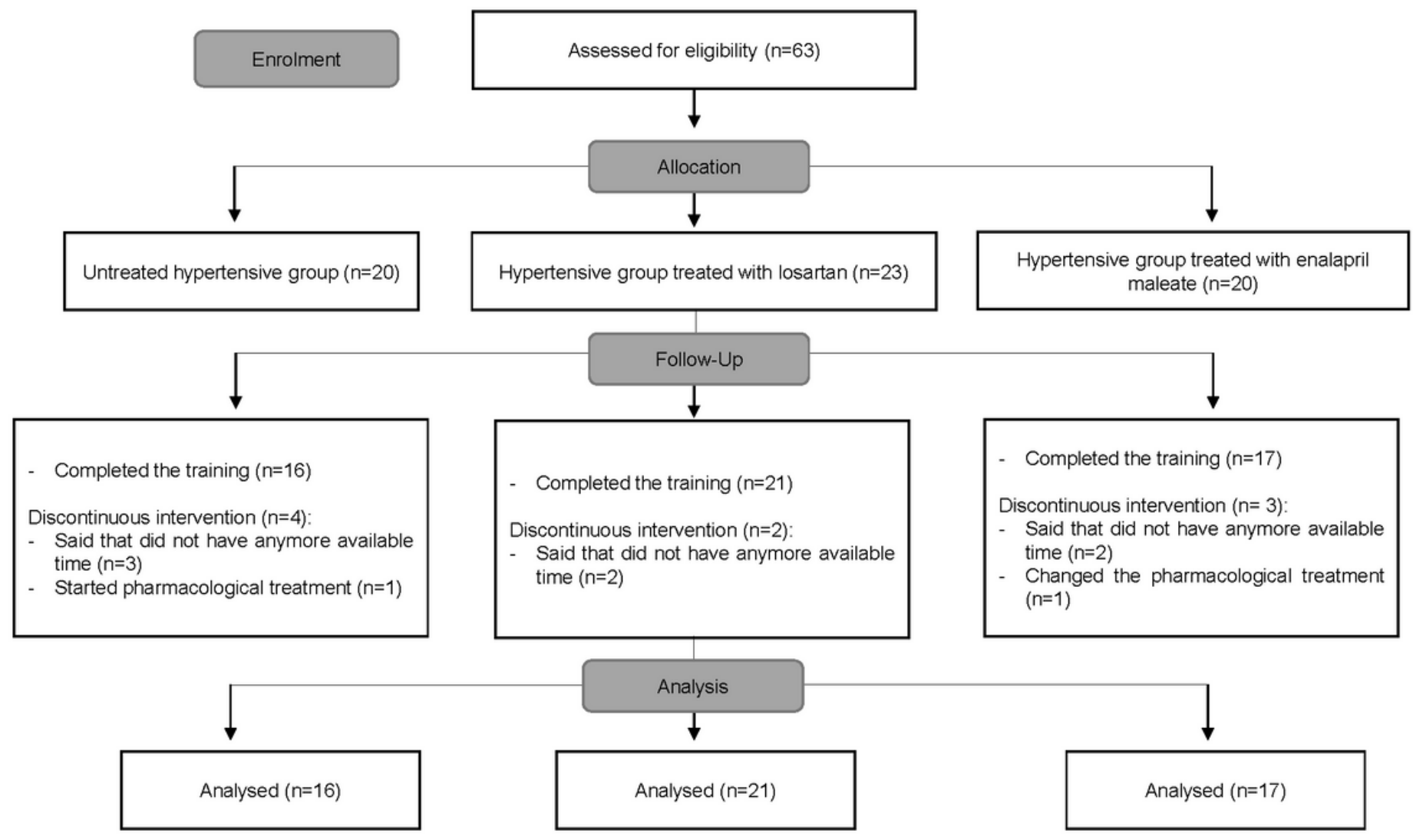

Figure 1

Flow diagram of participants through each stage of the study. 


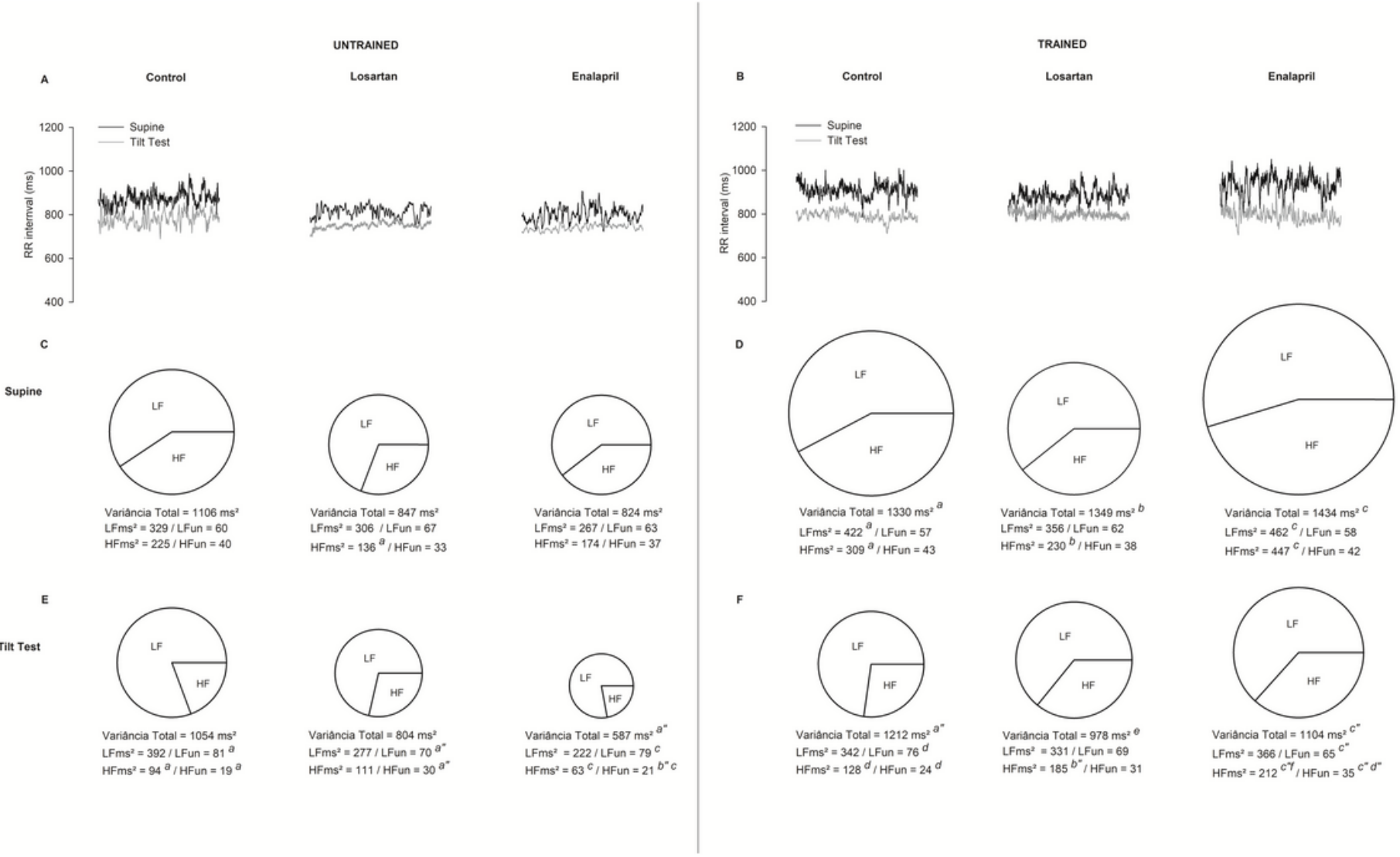

Figure 2

The RR interval time series from each group (i.e., tachograms) are illustrated in the top panels (A and B). Spectral analysis of HRV in hypertensive men at rest ( $C$ and $D$ ), during the active test tilt ( $E$ and $F$ ), and before ( $C$ and $E$ ) and after ( $D$ and $E$ ) aerobic physical training. The use of normalized units (nu) clearly indicates the altered relationship between LF and HF during the tilt test, as represented by the pie chart, which shows the relative distribution together with the absolute power of the two components represented by the area. Values are presented as the mean. $\mathrm{P} \leq 0.05$ versus ${ }^{a}$ Control supine; ${ }^{b}$ Losartan supine; ${ }^{c}$ Enalapril supine; ${ }^{d}$ Control after training supine; ${ }^{e}$ Losartan after training supine; ${ }^{f}$ Enalapril after training supine; ${ }^{a "}$ Control tilt test; $b^{\prime \prime}$ Losartan tilt test; ${ }^{c "}$ Enalapril tilt test; ${ }^{d "}$ Control after training tilt test.

\section{Supplementary Files}

This is a list of supplementary files associated with this preprint. Click to download.

- Supplementarylnformation.pdf 